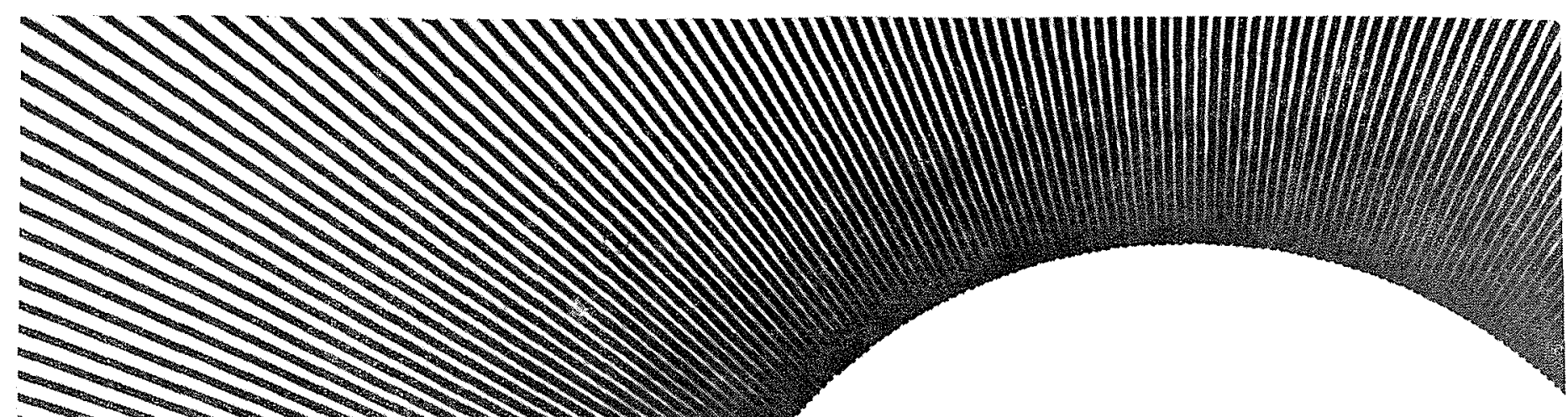

SOCIETE HYDROTECHNIQUE DE FRANCE

Prix Henri Milon 1972

\title{
L'ÉROSION DES SOLS PAR L'EAU EN CLIMAT MÉDITERRANÉEN. MÉTHODES EXPÉRIMENTALES POUR L'ÉTUDE DES QUANTITÉS ÉRODÉES A L'ÉCHELLE DU CHAMP
}

\author{
par J.-M. MASSON \\ Maitre Assistant au Laboratoire d'hydrologie \\ de l'Université des sciences et techniques \\ du Lanquedoc. Montpellier II
}

Ce texte résume une thèse de docteur-ingénieur soutenue le 2 juin 1971 à l'Université des sciences et techniques du Languedoc.

Cette étude de l'érosion a débuté avec l'année 1960. La jeune République tunisienne, soucieuse de préserver son patrimoine foncier, multipliait alors les actions de conservation des eaux et du sol (C.E.S.), suivant en cela les conseils de quelques agronomes et forestiers français comme Bugeat, Montjauze et Saccardy.

Compte tenu de l'importance des sommes investies dans les opérations anti-érosives, et aussi du manque de formation des cadres chargés de concevoir les projets, le Centre de Recherche et d'Expérimentation de Génie Rural

\section{L'équation universelle de perte de sol}

Cette équation énumère et combine les principaux facteurs de l'érosion qui sont:

- la pluie;

- la nature du sol;

- la pente (degré d'inclinaison et longueur);

- la végétation et les pratiques culturales;

- les pratiques conservatrices éventuelles (essentiellement les cultures en bandes alternées et les banquettes en courbes de niveau).

En dépouillant les nombreuses mesures d'érosion faites aux Etats-Unis, Wischmeier a constaté que, si tous les fac- teurs de l'érosion sont maintenus constants à l'exception de la pluie, l'érosion spécifique $A$ (poids de terre sèche par unité de surface) est proportionnelle à un indice d'agressivité $R$ calculé à partir des caractéristiques de pluie :

$$
A=K \cdot R
$$

Sur des sols de nature différente, les autres facteurs de l'érosion étant maintenus constants à l'exception de la pluie, le coefficient $K$ varie avec les types de sol et caractérise leur érodibilité.

Comme son nom l'indique, $\mathrm{R}$ n'est qu'un indice sans dimension obtenu, pour une pluie, à partir du produit de l'énergie de cette pluie par son intensité maximale en $30 \mathrm{mn}$.

Le système de Wischmeier, établi à partir de grandeurs mesurées en unités anglo-saxonnes, doit être transposé aux 
unités du système métrique. La transposition que nous avons adoptée conduit à calculer l'indice $\mathrm{R}$, à partir de l'énergie $\mathrm{E}$ de la pluie exprimée en joules par mètre carré et de son intensité maximale en $30 \mathrm{mn} \mathrm{I}_{30}$ exprimée en millimètres par heure, au moyen de l'expression:

$$
R=\frac{E \cdot I_{30}}{685}
$$

Dans ces conditions, l'indice d'agressivité $R$ obtenu est 2,5 fois plus élevé que l'indice américain, mais les érodibilités des sols sont comparables et le produit de l'indice d'agressivité par l'érodibilité donne directement des tonnes par hectare.

Pour prendre en compte la variabilité de chacun des autres facteurs de l'érosion, Wischmeier les a considérés isolément. Toutes les autres conditions étant identiques par ailleurs, il a calculé les valeurs du rapport: Erosion spécifique pour une valeur quelconque du facteur considéré, sur l'érosion spécifique pour une valeur de référence de ce même facteur.

Ces proportions, il les a supposées constantes et indépendantes des valeurs prises par les autres facteurs, ce qui est une hypothèse simplificatrice mais pratique.

Il a donné ainsi les valeurs susceptibles d'être prises par trois rapports concernant respectivement :

- la longueur de pente et le degré d'inclinaison, de symbole L.S;

- la végétation, de symbole C;

- les pratiques conservatrices, de symbole $\mathrm{P}$.

Les valeurs de référence du système sont:

- pour L.S, une pente de $9 \%$ et de $22 \mathrm{~m}$ de long;

- pour $\mathrm{C}$, une jachère continuellement travaillée;

- pour $\mathrm{P}$, le travail du sol dans le sens de la pente.

En définitive, l'équation universelle, qui permet d'estimer l'érosion produite par une pluie sur n'importe quel champ cultivé, s'écrit:

$$
A=K \cdot R \cdot L \cdot S \cdot C \cdot P
$$

Cette équation a été établie aux Etats-Unis à partir des résultats de milliers d'observations.

En Tunisie, il n'était pas question de nous livrer à une vérification rigoureuse de la formule, mais simplement de nous assurer que son emploi était possible. Dans l'affirmative, il fallait nous donner les moyens d'évaluer quantitativement les facteurs de l'érosion au moins dans les régions du pays qui y sont le plus sensibles.

La cartographie de l'agressivité des pluies devait faciliter la recherche des régions les plus sensibles à l'érosion.

\section{Cartographie de l'agressivité des pluies}

L'indice d'agressivité se calcule pluie par pluie sur chaque station où des enregistrements de pluviographe à rotation quotidienne sont disponibles.

Six heures sans pluie séparent deux pluies.

Si l'évaluation de l'intensité maximale en $30 \mathrm{mn}$ ne pose pas de problèmes, il n'en va pas de même du calcul de l'énergie.

L'énergie $E$ d'une pluie est en principe obtenue par la relation classique :

$$
\mathrm{E}=1 / 2 \mathrm{M} \cdot \mathrm{V}^{2}
$$

Si la pluie était formée de gouttes de même diamètre tombant à la même vitesse, cette relation serait facile à appliquer.

En fait, la pluie est formée de gouttes de diamètres différents. La proportion des gouttes d'un diamètre donné est fort variable. Selon leur diamètre, les gouttes arrivent au sol avec une vitesse différente.

Il faut donc appliquer la relation classique aux gouttes d'un même diamètre et faire la somme des résultats obtenus pour tous les diamètres rencontrés, en pondérant les résultats de chaque diamètre par la proportion des gouttes correspondantes dans la masse totale de la pluie.

On connaît bien la vitesse des gouttes en fonction de leur diamètre, mais il n'existe pas de moyen commode pour mesurer dans une pluie la proportion de gouttes correspondant à un diamètre donné. Cette proportion est en partie, mais en partie seulement, liée à l'intensité de la pluie. Aussi Wischmeier a-t-il pu établir une relation donnant directement l'énergie moyenne de la pluie à partir de son intensité. Cette relation s'écrit:

$$
E=8,73 \log I+11,9
$$

et donne des joules par mètre carré et par millimètre de pluie quand I est exprimé en millimètres par heure.

A l'aide de cette relation, après avoir fractionné la pluie en hauteurs de même intensité, il est facile de calculer les énergies de chaque fraction, puis de sommer.

De cette manière on calcule l'indice d'agressivité de chaque pluie, puis les indices d'agressivité mensuels et annuels, enfin l'indice d'agressivité moyen annuel pour toutes les stations équipées d'un pluviographe à rotation quotidienne.

Pour estimer la valeur de l'indice d'agressivité aux stations qui disposent seulement d'un pluviomètre, on utilise une relation de la forme:

$$
\mathrm{R}=a \mathrm{C}^{b}
$$

La variable $C$ est obtenue en faisant le produit de trois caractéristiques pluviométriques:

- la hauteur moyenne des précipitations annuelles;

- la hauteur de la pluie journalière de période de retour deux ans;

- la hauteur de l'averse de $1 \mathrm{~h}$ et de période de retour deux ans.

Les coefficients $a$ et $b$, spécifiques d'une région, sont calculés à partir des points où l'indice d'agressivité est connu.

Enfin, tout autant que sa valeur absolue, c'est la répartition saisonnière de l'indice d'agressivité, qui, en faisant coincider des pluies très agressives avec un sol non protégé par les cultures, favorise l'érosion.

En Tunisie, compte tenu du fait que la région du nordouest est couverte de forêts, c'est la région de Pont-du-Fahs qui est l'une des plus sensibles à l'érosion. C'est là que nous avons mis en cuvre l'essentiel de nos dispositifs expérimentaux.

dispositifs expérimentaux

Ils consistent essentiellement :

- en parcelles fixes de mesure de l'érosion;

- en un simulateur de pluie;

- en parcelles fixes de mesure du rendement des cultures. 


\section{Les parcelles fixes de mesure de l'érosion.}

Le principe en est simple: Des bordures étanches délimitent une parcelle au bas de laquelle un dispositif recueille les eaux de ruissellement qui transportent la terre érodée.

Plutôt que de recueillir la totalité du mélange boueux, ce qui nécessite de grosses installations, nous avons choisi d'en recueillir une fraction connue.

Simples en théorie, ces principes ont fait surgir de nombreuses difficultés pratiques dans leur mise en cuvre.

La mesure des quantités érodées après chaque pluie est effectuée selon un protocole rigoureux, tandis que les précipitations sont mesurées par un pluviographe situé à proximité.

Les résultats d'une parcelle isolée sont difficilement utilisables, aussi les parcelles sont groupécs et soumises à un plan d'expérience.

Un premier groupe de deux fois trois parcelles de longueur différente, disposées de façon à permettre une analyse de variance en blocs complets, ne nous a pas permis de mettre en évidence l'influence de la Iongueur de pente sur l'érosion. Ramenées toutes à la même longueur et conduites de façon identique, ces parcelles nous ont ensuite permis de voir que l'indice d'agressivité était l'une des caractéristiques de pluie les mieux correlées à l'érosion.

Un autre groupe de dix parcelles nous a servi à tester l'infiuence de la végétation et des pratiques culturales sur l'érosion.

\section{Le simulateur de pluie.}

Les parcelles fixes de mesure de l'érosion sont des dispositifs coûteux, liés à un type de sol et soumis au caprice du temps quant à leurs résultats.

Pour obtenir rapidement de nombreuses mesures sur différents types de sol, nous avons été amenés à construire et à utiliser un simulateur de pluie.

Un tel dispositif n'est valable qu'à la condition de pouvoir comparer la pluie simulée aux pluies naturelles. L'indice d'agressivité permet cettc comparaison.

Des ajutages de fabrication industrielle, espacés de $0,50 \mathrm{~m}$ dans un sens et de 1,60 $\mathrm{m}$ dans l'autre, disposés à $2,40 \mathrm{~m}$ de hauteur sur des portiques pliants haubannés, et alimentés alternativement à partir d'un camion-citerne par une motopompe, nous ont permis de reproduire sur une parcelle $12,5 \mathrm{~m}^{2}$ en projection verticale une pluie suffisamment homogène qui, appliquée pendant un quart d'heure fournit un indice d'agressivité comparable à celui d'une pluie de période de retour quinze ans pour la région de Pont-du-Fahs.

L'eau boueuse qui ruisselle est recueillie et l'érosion mesurée selon un certain protocole.

$\mathrm{Ce}$ dispositif, avec lequel deux cent cinquante-quatre essais ont été effectués, nous a permis entre autres choses de déterminer l'érodibilité des sols.

\section{Les parcelles fixes de rendement des cultures.}

Semblables à tous les dispositifs expérimentaux utilisés en agriculture, elles nous ont permis de mesurer l'influence que pouvaient avoir sur les rendements des procédés culturaux efficaces contre l'érosion.

Ces parcelles étaient réparties sur trois périmètres expérimentaux :

- un périmètre situé en terrain plat;

- un périmètre situé sur un terrain avec une pente de $5 \%$;

- un périmètre réservé plus spécialement à la comparaison traction mécanique - traction animale.
Détermination de l'érodibilité des sols

Ce sont de nombreux essais effectués au simulateur de pluie, dans des conditions de pente voisines et avec une couverture végétale identique (chaumes de céréales), qui ont permis cette détermination.

Un tableau de l'érodibilité des principaux types de sol de la région de Pont-du-Fahs a été dressé. Cette érodibilité varie de 1 à 12

Dumas a utilisé les résultats obtenus pour établir une relation entre les valeurs de l'érodibilité $\mathrm{K}$ et certaines caractéristiques analytiques du sol. Il a abouti à l'expression:

$$
\begin{aligned}
\log 1000 \mathrm{~K}= & 3,4623-0,1695 \text { mo }-0,0214 \mathrm{He} \\
& 0,0282 \text { cailloux }
\end{aligned}
$$

ajustée sur trente points avec un coefficient de corrélation linéaire multiple de 0,94 .

Les caractéristiques analyiiques sont mesurées sur un mélange d'échantillons de la surface du sol $(5 \mathrm{~cm}$ de profondeur) séché à l'air. Ce sont:

- le pourcentage de refus au tamis de $2 \mathrm{~mm}$ (variable cailloux);

- le taux de matière organique (variable mo);

- l'humidité équivalente (variable $\mathrm{He}$ ).

\section{Influence des cultures et du mode de travail du sol}

\section{Protection apportée par les assolements.}

En matière d'agriculture, les états de la surface du sol se succèdent au rythme des saisons et des travaux agricoles. Il y a des périodes vù le sul est la ès vuluérable, l'autres au contraire où il est bien protégé, et ces périodes peuvent coïncider ou non avec la saison où se produisent habituellement les pluies les plus agressives.

Un assolement couvre plusieurs années. Chaque année est caractérisée par une succession de difiérents états de la surface du sol. Il faut déterminer la valeur du facteur $\mathrm{C}$ correspondant à chacun de ces états, puis sommer ces valeurs en les pondérant par le pourcentage de l'indice d'agressivité moyen annuel qui se produit pendant la période correspondante. La valeur moyenne du facteur $C$ pour un assolement est ainsi une valeur régionale qui dépend de la répartition moyenne dans le temps des pluies érosives et de la date des travaux agricoles.

On distingue en général cinq périodes culturales de sensibilité différente:

- la période des labours grossiers exécutés avant toute culture;

- la période correspondant à la préparation de la terre pour le semis et au semis proprement dit;

- la période d'établissement de la végétation;

- la période de végétation;

- la période qui s'étend de la récolte au labour grossier suivant.

Des expérimentations faites au simulateur de pluie et sur les parcelles fixes nous ont permis de montrer, sur quelques périodes culturales particulières, la bonne concordance des valeurs trouvées pour le facteur $C$, avec celles annoncées par les auteurs américains. 
En extrapolant certaines données à partir des tables du «Soil Conservation Service» des Etats-Unis, nous avons pu calculer les valeurs moyennes annuelles du facteur $C$ pour les assolements existants et ceux recommandés par les services agricoles tunisien.

La protection apportée par les cultures varie de 1 à 100 .

\section{Influence de pratiques agronomiques spéciales sur l'érosion.}

La sensibilité à l'érosion d'une culture dépend aussi du type d'instrument utilisé pour ameublir le sol. On distingue essentiellement :

- les instruments de culture habituels, qui retournent la terre;

- les instruments de culture sans retournement, qui ameublissent le sol en laissant en surface les débris de la récolte précédente.

Nous avons donc testé les répercussions, sur l'érosion, de l'emploi de ces deux types d'instruments.

Les résultats de deux essais du simulateur sur trois répétitions de six traitements, analysés par les techniques de l'analyse de variance, furent formels : Le travail sans retournement diminue l'érosion et favorise l'infiltration.

A la suite d'une expérimentation semblable sur parcelles fixes, nous avons également vérifié l'influence significative du type d'instrument utilisé sur les paramètres de la régression liant les quantités érodées à l'intensité maximale en $30 \mathrm{mn}$ de la pluie.

On en conclut que l'utilisation d'instruments de culture sans retournement à la place des appareils classiques double la protection apportée au sol pendant les périodes les plus vulnérables des assolements.

\section{influence de pratiques agronomiques spéciales} sur le rendement.

Bien que le travail du sol sans retournement réduise efficacement l'érosion, il faut s'assurer qu'il n'a pas une action nćfastc sur lc rendement des cultures avant de le resommander aux agriculteurs.

L'expérimentation a été conduite sur les parcelles fixes de rendement des cultures essentiellement au moyen de plans factoriels. Comme d'autres essais effectués par les services de l'agricuiture, nous avons montré que les techniques de culture sous résidus pouvaient donner des rendements comparables à ceux obtenus avec les techniques culturales habituelles à condition d'en limiter l'usage à la saison la plus dangereuse, de lutter efficacement contre les mauvaises herbes et de soigner les semis. Accessoirement on a montré aussi qu'en traction animale les rendements pouvaient rivaliser avec ceux obtenus en traction mécanique quelles que soient les techniques utilisées.

\section{La notion de tolérance de pertes en terre indispensable pour utiliser l'équation de perte du sol}

Maintenant que nous nous sommes donné les moyens d'évaluer quantitativement les facteurs de l'érosion dans la région de Pont-du-Fahs, nous pouvons rapidement et facilement, grâce à l'équation de perte de sol, estimer dans cette région l'érosion d'un champ soumis à une pluie donnée.

A l'aide de l'indice d'agressivité moyen annuel, nous pouvons aussi évaluer l'érosion moyenne annuelle de ce champ.
Mais une question importante restait en suspens: Comment l'équation universelle pouvait-elle nous aider à concevoir les aménagements anti-érosifs à mettre éventuellement en œuvre sur ce champ?

Ces aménagements ont pour but, non pas de supprimer l'érosion, mais de la ramener à un seuil tolérable, en agissant sur les facteurs contrôlables qui sont par ordre d'importance: la végétation, les pratiques conservatrices et la longueur de la pente.

Comment déterminer la valeur $\mathrm{T}$ au-dessus de laquelle l'érosion est dangereuse et en-dessous de laquelle elle est au contraire tolérable? Faute de mieux, nous nous sommes inspirés des normes américaines basées uniquement sur la profondeur pour déterminer combien de terre les différents types de sol rencontrés en Tunisie pouvaient perdre en moyenne par hectare et par an. Les limites s'établissent ainsi :

Sols minces $(<30 \mathrm{~cm}) \ldots \ldots 2,5 \mathrm{t} / \mathrm{ha} / \mathrm{an}$

Sols moyens $(30$ à $70 \mathrm{~cm}) \ldots \ldots 5,0 \mathrm{t} / \mathrm{ha} / \mathrm{an}$

Sols profonds $(>70 \mathrm{~cm}) \ldots \ldots 10,0 \mathrm{t} / \mathrm{ha} / \mathrm{an}$

Remarquons que $10 \mathrm{t} /$ ha correspondent annuellement à moins de $1 \mathrm{~mm}$ d'épaisseur.

Vis à vis de l'érosion, un sol est donc défini par deux caractéristiques :

- son érodibilité $\mathrm{K}$;

- sa tolérance $\mathrm{T}$.

Pour déterminer rapidement quelles valeurs il faut donner aux facteurs contrôlables pour ramener l'érosion au niveau $\mathrm{T}$, j'ai imaginé une règle coulissante basée d'une part sur la propriété qu'ont les logarithmes de transformer produits et rapports en additions et soustractions, et d'autre part sur des graduations fonctionnelles.

Cette règle a été construite. Elle était distribuée aux projeteurs tunisiens accompagnée des cartes et tableaux nécessaires pour transformer les facteurs de l'érosion en valeurs numériques, c'est-à-dire :

- une carte de l'indice d'agressivité moyen annuel $R$ sur la Tunisie;

- un tableau de correspondance entre les caractéristiques pédologiques des sols et la valeur du rapport $\mathrm{T} / \mathrm{K}$;

- un tableau de correspondance entre les couvertures végétales pérennes ou les assolements et la valeur du facteur $\mathrm{C}$.

Cette règle fonctionne de plusieurs manières :

- On peut se fixer l'assolement, donc la valeur du facteur C correspondante. Compte tenu des conditions locales (valeur de l'indice d'agressivité moyen annuel, valeur du rapport $T / K$, pourcentage de pente), la règle donne la longueur maximum de la pente qui peut être cultivée:

- sans pratiques conservatrices;

- en travaillant le sol en courbes de niveau;

- en appliquant la technique des bandes alternées.

Cette dernière longueur correspond à l'écartement horizontal des banquettes qui couperont la pente si cette dernière est trop longue.

- On peut aussi fixer une limite aux pratiques conservatrices, par exemple décider de ne pas construire de banquettes. Dans ce cas la règle donne les assolements qu'il est possible de cultiver dans ces conditions sans que l'érosion dépasse le seuil de tolérance.

De toute façon, la règle rappelle aux projeteurs expérimentés, qu'en matière de conservation des eaux et du sol, les solutions d'aménagement présentent souvent plusieurs combinaisons possibles, et que le choix de la meilleure résulte d'un calcul économique. 


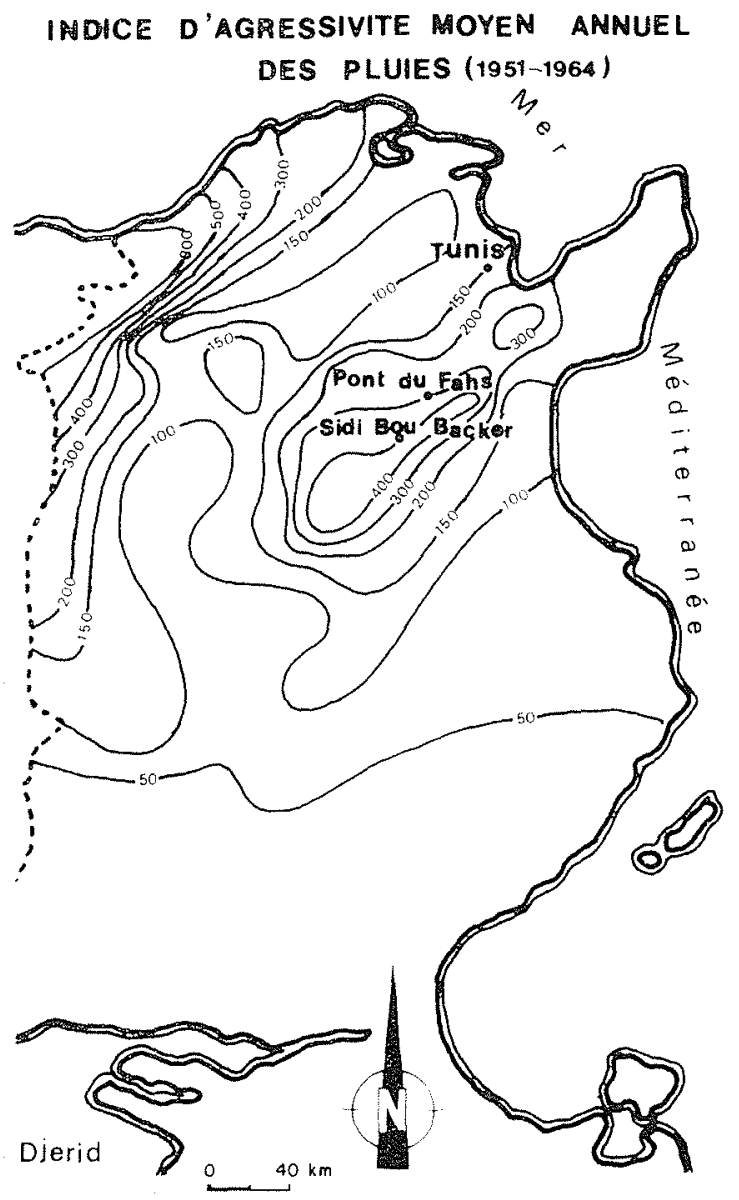

REPATTITION MOYENAE DE L'INDICE D'AGRESSIVITE (1951-1964) - insice d'sagrassivité
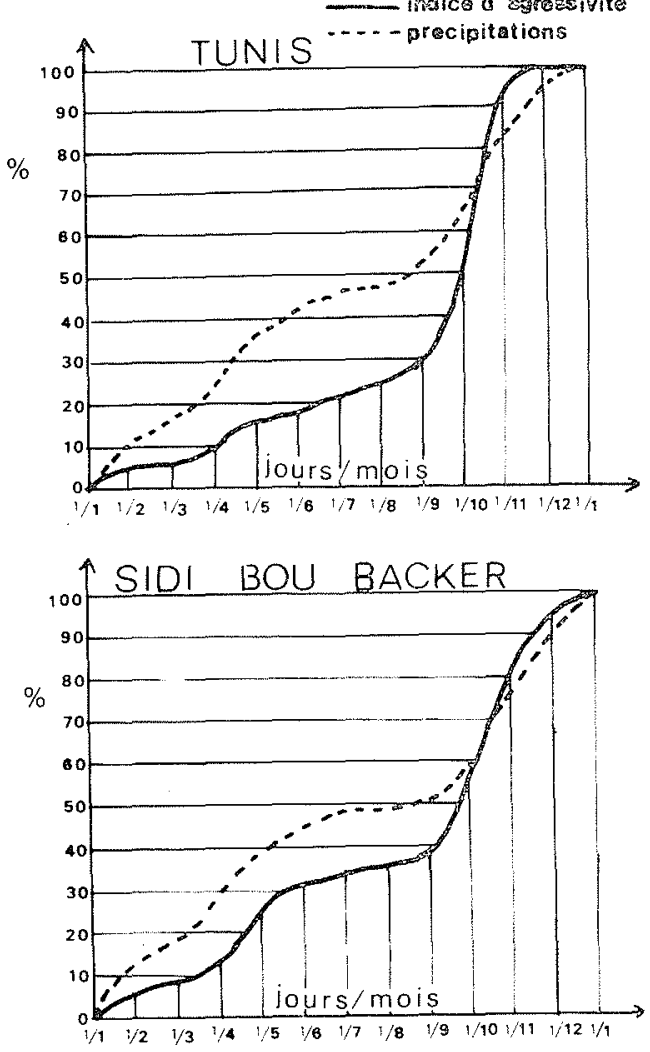

REgLE COULISSANTE FACILITANT L'ELABORATION DES FROJETS DE CONSERVATION DES EAUX ET DU SOL

LE CLIMAT ( $R$ ) ET LE SOL ( $T / K$ ) CONDITIONNENT LA GAMME DES COMBINAISONS: ASSOLEMENTS - PRATIQUES CONSERVATRICES

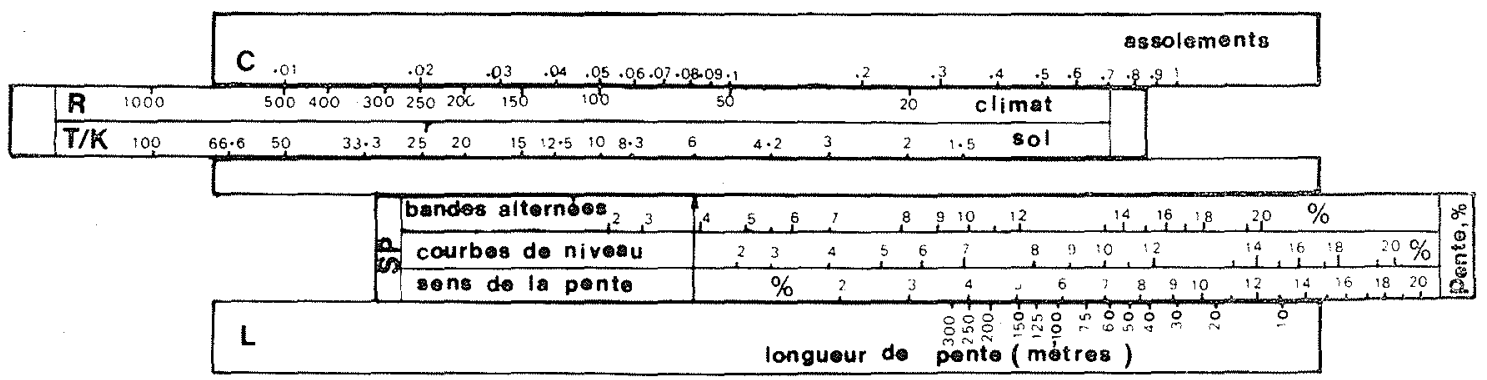

TYPE DE BANOUETTE POUR LES

CULTURES ARBUSTIVES
TYPE DE BANQUETTE POUR LES

CULTURES HERBACEES
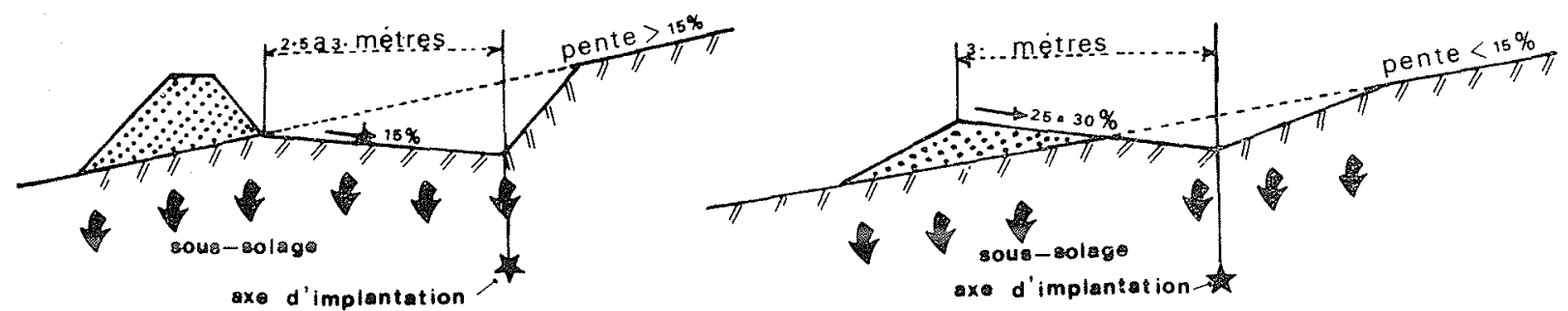


\section{Application des résultats aux projets}

Méthodologie pour l'aménagement d'une exploitation agricole.

A cette échelle, l'indice d'agressivité moyen annuel prend une seule valeur pour toute la surface et l'objectif est uniquement d'assurer la pérennité de l'agriculture.

Les documents de base nécessaires sont une carte pédologique à grande échelle et les photographies aériennes, sur lesquels on reportera le projet car chaque point du territoire y est facilement repérable par le projeteur et les équipes d'implantation.

On cartographie tout d'abord les zones homogènes quant aux caractéristiques du sol (rapport $\mathrm{T} / \mathrm{K}$ ) et au pourcentage de pente.

Sur chaque zone, la carte d'aptitude aux cultures qui, en Tunisie, accompagne chaque carte pédologique, donne les assolements possibles.

A ces différents assolements, correspondent des pratiques conservatrices différentes qu'on détermine sur la règle compte tenu de la valeur du facteur $\mathrm{C}$ relatif à chaque assolement. On peut donc pour chaque assolement, associer le revenu des cultures au coût des pratiques conservatrices.

Choisir un couple assolement-pratiques conservatrices, c'est choisir un investissement actuel plus ou moins élevé et un revenu futur plus ou moins important.

Le choix ne peut se faire qu'à partir d'options économiques. Parmi les spéculations possib'es sera choisie celle qui, ayant à amortir et aussi à entretenir les pratiques conservatrices. donnera la plus forte valeur au paramètre économique choisi (revenu net, valeur ajoutée, salaires, etc.).

Le choix étant fait, le projeteur pourra alors exercer tout son art pour délimiter des parcelles culturales de dimension commode et d'accès facile. pour choisir les voies d'eau qui serviront d'exutoire et celles qui seront neutralisées et enfin pour représenter le tout sur un document cartographique simple et lisible.

\section{Méthodologie pour l'aménagement d'un bassin versant (plusieurs milliers de $\mathrm{km}^{2}$ ).}

II s'agit de déterminer:

- les principales options à prendre;

- les crédits à dégager:

- les zones prioritaires.

\section{CHOIX des optIons et dÉTERMination des Coûts.}

Les documents indispensables sont les cartes pédologiques et topographiques au $1 / 50000$ et les photographies aériennes récentes.

Le premier travail consiste à faire l'inventaire des facteurs de l'érosion et des possibilités agrico'es. La meilleure façon d'opérer consiste à procéder à un échantillonnage par points. Le traitement des résultats à l'ordinateur permet d'envisager un point tous les demi-centimètres au 1/50000. soit seize points au $\mathrm{km}^{2}$.

Chaque point, repéré par ses coordonnées Lambert, est caractérisé :

- par les cultures qu'il est possible d'y faire;

- par les résultats économiques de ces cultures;

- par le coût des pratiques conservatrices qu'il faut y associer pour que les résultats économiques durent.

Le choix se fait bien sûr en fonction d'un paramètre économique choisi au départ. On peut ensuite évaluer le coût de l'ensemble et la proportion de chaque type d'aménagement.

\section{CHOIX DES PRIORITÉS.}

Se plaçant d'un point de vue agricole, une méthode consiste à comparer dans des conditions d'érosion tolérable la meilleure situation économique sans C.E.S. avec celle permise après travaux C.E.S.

La priorité est donnée là où les différences sont les plus fortes, cette différence portant bien entendu sur un critère économique à déterminer. On peut rapporter les différences aux sommes à investir et définir un intérêt relatif.

Quand la construction de nombreux barrages collinaires est prévue, on peut déterminer une priorité dans le traitement des bassins versants des barrages.

Grâce à nos points échantillons nous pourrons estimer la terre arrachée annuellement au bassin versant du barrage dans différentes conditions. L'importance relative de l'envasement pourra être chiffirée par la valeur du rapport:

Terre arrachée annuellement au bnssin versant du barrage sur le volume de la retenue.

Plus ce rapport est élevé, plus le barrage s'envase vite. On peut ainsi classer les barrages.

Pour mesurer l'intérêt du traitement du bassin versant du barrage, on peut utiliser l'expression:

$$
\frac{\left(A_{1}-T\right) P / V}{2 D}
$$

avec :

$A_{1}$ : terre arrachée sur le bassin versant dans les conditions actuelles;

$\mathrm{T}$ : terre arrachée sur le bassin versant après traitement (égale ou inférieure à la tolérance);

$P$ : coût du barrage à amortir sur $2 n$ années;

D : coût du traitement C.E.S. à amortir sur $n$ années;

$\mathrm{V}$ : volume de la retenue.

Plus l'expression prend une valeur élevée, plus le bassin versant du barrage est intéressant à traiter.

\section{Conclusion}

L'équation universelle de perte de sol nous a fourni au départ les hypothèses de travail nécessaires à une étude sur l'érosion, d'abord pour identifier ses principaux facteurs, ensuite pour déterminer la manière dont ils se combinent pour produire leurs effets.

Pendant plusieurs années nous avons confronté les hypothèses aux faits. Nous avons utilisé pour ce faire des dispositifs expérimentaux et certaines techniques mathématiques et statistiques.

Nous pensons avoir déterminé, avec une approximation suffisante pour l'usage qu'il est intéressant d'en faire, les conditions d'application en Tunisie de ce modèle d'érosion cur'est l'équation universelle de perte de sol. Son intérêt tient surtout au fait qu'elle permet d'estimer l'érosion non seulement dans les conditions actuelles d'exploitation du sol, mais aussi dans n'importe quelle autre condition.

Pour résoudre rapidement les problèmes posés techniquement par le choix des aménagements anti-érosifs, nous avons imaginé un dispositif comparable à celui d'une règle à calcul. Il ne faut voir dans cette règle coulissante qu'un moyen commode pour obtenir des ordres de grandeur et pour concevoir des projets qui prennent en compte les facteurs de l'érosion d'une même manière objective, basée sur les résultats de mesures.

Pour nous, le progrès ne consiste pas à obtenir des résultats expérimentaux, mais à rendre l'expérience transmissible pour qu'elle ne reste pas, selon le mot d'un auteur, «La lanterne qui n'éclaire que celui qui la porte». 